\title{
Stenotic Transverse Sinus Predisposes to Poststenting Hyperperfusion Syndrome as Evidenced by Quantitative Analysis of Peritherapeutic Cerebral Circulation Time
}

\author{
C.-J. Lin, F.-C. Chang, F.-Y. Tsai, W.-Y. Guo, S.-C. Hung, D.Y.-T. Chen, C.-H. Lin, and C.-Y. Chang
}

\begin{abstract}
BACKGROUND AND PURPOSE: Hyperperfusion syndrome is a devastating complication of carotid stent placement. The shortening of cerebral circulation time after stent placement is seen as a good indicator of the development of hyperperfusion syndrome. The purpose of our study was to evaluate whether patients with ipsilateral transverse sinus stenosis are prone to having shortened cerebral circulation time after stent placement, subsequently leading to the possible development of hyperperfusion syndrome.
\end{abstract}

MATERIALS AND METHODS: Forty-nine patients with $>70 \%$ unilateral carotid stenosis undergoing stent placement were recruited for analysis. Group A consisted of patients with a stenotic ipsilateral transverse sinus $>50 \%$ greater than the diameter of the contralateral transverse sinus; the remaining patients were in group B. Quantitative DSA was used to calculate cerebral circulation time. Cerebral circulation time was defined as the time difference between the relative time to maximal intensity of ROIs in the proximal internal carotid artery and the internal jugular vein. $\Delta C C T$ was defined as cerebral circulation time before stent placement minus cerebral circulation time after stent placement. $\Delta \mathrm{CCT}$, white matter hyperintensity signals, and sulcal effacement in MR imaging were compared between the 2 groups.

RESULTS: $\triangle C C T$ was significantly shorter in group A $(0.65 \pm 1.3)$ than in group B $(-0.12 \pm 1.4)$. Three patients had white matter hyperintensity signals in group A, and 1 developed hyperperfusion syndrome. Group B showed no MR imaging signs of hyperperfusion syndrome.

CONCLUSIONS: Ipsilateral hypoplastic transverse sinus was associated with prolonged cerebral circulation time before stent placement and greatly shortened cerebral circulation time after stent placement. Inadequate venous drainage might play a role in impaired cerebral autoregulation and might influence the development of poststenting hyperperfusion syndrome.

ABBREVIATIONS: $C C T=$ cerebral circulation time; $\triangle \mathrm{CCT}=\mathrm{CCT}$ after stent placement minus $\mathrm{CCT}$ before stent placement; $\mathrm{HPS}=$ hyperperfusion syndrome; $\mathrm{TS}=$ transverse sinus

$C^{a}$ arotid stent placement restores cerebral perfusion to the ischemic area to prevent further ischemic insults. The normalized cerebral circulation after stent placement also correlates with improved neurologic functional outcomes. ${ }^{1-3}$ Although carotid stent placement has been proved a safe therapy for those with significant extracranial stenosis, complications do occur, includ-

Received August 4, 2013; accepted after revision October 17

From the Department of Radiology (C.-J.L., F.-C.C., W.-Y.G., S.-C.H., C.-Y.C.), Taipei Veterans General Hospital, Taipei, Taiwan; School of Medicine (C.-J.L., F.-C.C., W.-Y.G., S.-C.H., C.-Y.C.), National Yang Ming University, Taipei, Taiwan; Imaging Research Center (F.-Y.T., D.Y.-T.C.), Taipei Medical University, Taipei, Taiwan; Department of Medical Imaging (D.Y.-T.C.), Shuang-Ho Hospital, New Taipei City, Taiwan; and Graduate Institute of Epidemiology and Preventive Medicine (C.-H.L.) National Taiwan University, Taipei, Taiwan.

This work was cosponsored by Taipei Veterans General Hospital and Siemens (grant number T1100200)

Please address correspondence to F.-Y. Tsai, MD, FACR, Imaging Research Center, Taipei Medical University, $250 \mathrm{Wu}$-Hsing St, Taipei, 11031, Taiwan; e-mail:

ftsai@uci.edu or ftsai@tmu.edu.tw

http://dx.doi.org/10.3174/ajnr.A3838 ing thromboembolic events and hyperperfusion syndrome. ${ }^{4} \mathrm{Hy}-$ perperfusion syndrome has a characteristic triad (headache, seizure, and focal neurologic deficits) and typically occurs within several hours or days after the procedure. ${ }^{5-7}$ Risk factors for hyperperfusion have been proposed, including high-grade stenosis, poor collateral circulation, high blood pressure, age, and bloodbrain barrier breakdown. ${ }^{7-11}$

Nevertheless, the underlying pathophysiology of HPS has not been well evaluated due to its low incidence $(0.7 \%-3 \%))^{7,9,12}$ The generally accepted explanation emphasizes a failure of autoregulation after sudden augmentation of cerebral blood inflow following carotid stent placement. To assess cerebral perfusion objectively and immediately within the angiography suite, we used quantitative color-coded DSA to evaluate the changes in cerebral circulation time before and after the procedures. ${ }^{13,14}$ CCT is defined as the time required for blood to traverse through the brain, and it can serve as a surrogate for cerebral perfusion during angiography. ${ }^{15}$ Obtaining immediate cerebral hemodynamic mea- 
surements can help detect HPS early and facilitate immediate, aggressive postprocedural blood pressure control.

Despite careful patient selection and postprocedural care, we have nonetheless encountered unexpected cases of HPS after carotid stent placement. A recent study has shown that shortened CCT after carotid stent placement carries higher risks for HPS. ${ }^{12}$ After reviewing our cases, we found that those patients with HPS usually had ipsilateral hypoplastic or atretic transverse sinuses. The purpose of our study was to determine the impact of venous stenosis on CCT after stent placement and to determine whether venous stenosis is a risk factor for HPS.

\section{MATERIALS AND METHODS \\ Patient Selection}

We retrospectively reviewed 60 patients who underwent carotid stent placement from June 2011 to December 2012 in our hospital. The inclusion criterion for this study was having unilateral carotid stenosis of $>70 \%$ according to the NASCET criteria. Three patients with tandem intracranial stenosis or contralateral extracranial stenosis were excluded; 2 cases of dissection and 2 cases with suboptimal DSA imaging quality due to motion artifacts were also excluded. Respiratory problems or congestive heart failure can also alter the waveforms and subsequent CCTs, so we also excluded 4 patients with known heart disease. Fortynine patients were available for analysis and were divided into 2 groups according to the caliber of the ipsilateral transverse sinus based on measurements taken from their DSA images. Group A consisted of patients with hypoplastic TS (degree of stenosis at least $50 \%$ greater than on the contralateral side) or atretic TS (nonmeasurable, with stenosis assumed to be total). Group B consisted of patients with no stenosis (the degree of stenosis identical to that of the contralateral TS) or mild narrowing (greater degree of stenosis than on the contralateral side but $<50 \%$ greater). Before assessing CCT, 1 neuroradiologist objectively measured the degree of stenosis on the anteroposterior view when the TS was optimally visualized. HPS was defined as abnormally high signal intensity involving periventricular or subcortical white matter or sulcal effacement on FLAIR sequences of posttreatment MR imaging, in combination with ipsilateral headache with or without nausea and vomiting, focal seizure, and neurologic deficits. ${ }^{9}$

\section{Peri-Carotid Stent Placement Management}

All patients were premedicated with aspirin, $100 \mathrm{mg} /$ day, and clopidogrel, $75 \mathrm{mg} /$ day, for at least 2 weeks before stent placement. Stent placement was performed with the patient under general anesthesia. Intraoperative heparin was given to maintain an activated thrombin time of $>200$ seconds. The end point of the successful procedure was defined as residual stenosis of $<10 \%$. The patients were transferred to a stroke intensive care unit for strict blood pressure control below $130 \mathrm{~mm} \mathrm{Hg}$ for 24 hours.

\section{DSA}

Quantitative color-coded cerebral angiography was performed to measure changes in cerebral circulation time during carotid stent placement between the 2 groups. The procedures were performed by using 1 flat panel angiography system (Axiom Artis; Siemens,

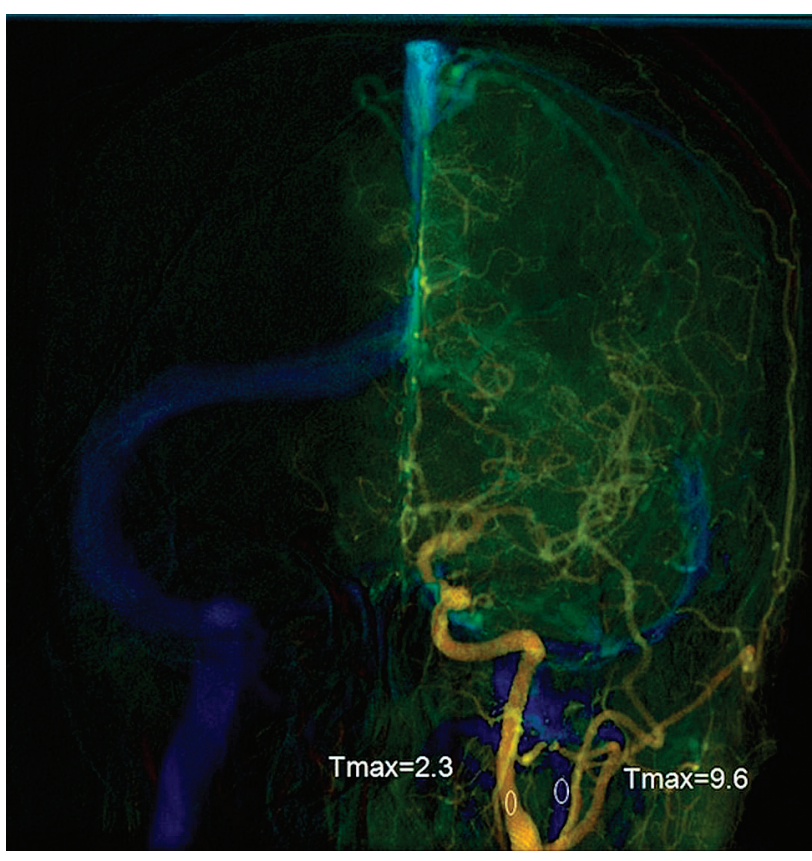

FIG 1. Anteroposterior view of quantitative brain DSA of a 51-yearold woman. The midpoint of the cervical portion of the internal carotid artery was selected for the arterial region of interest. The midpoint of the ipsilateral internal jugular vein was selected for venous region of interest. Cerebral circulation time was the time difference between the 2 ROIs.

Erlangen, Germany). A 4F angiocatheter was placed at the level of the $\mathrm{C} 4$ vertebral body for DSA. The fixed acquisition protocol was performed on all patients according to Lin et al. ${ }^{13}$ All patients were told to breath-hold during the DSA acquisition. Postprocessing software (syngo iFlow; Siemens) was used to quantify the selected ROIs according to their time to maximal intensity in seconds. ${ }^{14}$ The time to maximal intensity of any selected region of interest was defined as the time point when the attenuation of the $\mathrm{x}$-ray reached its maximum along the angiographic frames. An anteroposterior view was used to determine CCT. It was defined as the difference in time to maximal intensity between arterial ROIs in the proximal internal carotid artery and the internal jugular vein (Fig 1).The diameters of all ROIs were intended to cover the calibers of the selected vessels without overlapping other vessels. $\triangle \mathrm{CCT}$ is equal to CCT after stenting minus CCT before stenting.

\section{MR Imaging}

All peritherapeutic MR imaging was conducted in the same scanner (Signa Excite HD; GE Healthcare, Milwaukee, Wisconsin). Prestenting MRA with time-of-flight technique (TR $=28 \mathrm{~ms}$, $\mathrm{TE}=7 \mathrm{~ms}$, flip angle $=20^{\circ}$, matrix size $\left.=384 \times 224\right)$ was used to assess collaterals. Lack of collateral circulation was considered as the absence of either the anterior or posterior communicating artery according to the original reports. Selective angiography does not always demonstrate the anterior and posterior cerebral arteries well because of the competing flow from contralateral noniodinated blood flow. This phenomenon is more profound when there is an ipsilateral proximal carotid stenosis. FLAIR sequences $(\mathrm{TR}=9000 \mathrm{~ms}, \mathrm{TE}=120 \mathrm{~ms}$, $\mathrm{TI}=2250 \mathrm{~ms}$, section 
thickness $=5 \mathrm{~mm}$, matrix size $=288 \times 224$ ) of the brain before and after stent placement were used to compare the white matter changes and the gyral effacement, by the consensus of the same 2 experienced neuroradiologists who analyzed the DSA datasets, but in different sessions.

\section{Statistics}

The $\Delta$ CCT between 2 groups was analyzed by using $t$ tests. The white matter changes and incidences of HPS were determined by using the Fisher exact test. $P<.05$ was considered significant. Statistical analysis was performed by using the Statistical Package for the Social Sciences, Version 20 (IBM, Armonk, New York). Diagnostic tests performed with the Youden index, and the ROCs for both prestent CCT and $\triangle \mathrm{CCT}$ versus HPS were evaluated.

Table 1: Patient characteristics between those with an ipsilateral stenotic transverse sinus (group A) and those with a normal transverse sinus (group B)

\begin{tabular}{lcc}
\hline & Group A $(\boldsymbol{n}=15)$ & Group B $(\boldsymbol{n}=\mathbf{3 4})$ \\
\hline Age $(\mathrm{yr})$ & $74.5+10.2$ & $73.9+10.3$ \\
Sex (M/F) & $12: 3$ & $31: 5$ \\
Hypertension & $5(33 \%)$ & $13(38 \%)$ \\
MR imaging evidence of & $6(40 \%)$ & $16(47 \%)$ \\
$\quad$ prior stroke & & \\
Stenosis degree & $82 \%$ & $81 \%$ \\
Lack of collaterals in MRA & $2(13 \%)$ & $6(18 \%)$ \\
\hline
\end{tabular}

Table 2: Peritherapeutic circulation times and MR imaging findings between those with an ipsilateral stenotic transverse sinus (group A) and those with a normal transverse sinus (group B)

\begin{tabular}{lccc}
\hline & $\begin{array}{c}\text { Group A } \\
(\boldsymbol{n}=15)\end{array}$ & $\begin{array}{c}\text { Group B } \\
(\boldsymbol{n}=34)\end{array}$ & $\boldsymbol{P}$ Value \\
\hline DSA & & & \\
$\quad$ Prestent CCT & $6.9 \pm 1.2$ & $6.2 \pm 1.1$ & .14 \\
$\quad \begin{array}{l}\text { Poststent CCT } \\
\quad \text { CCT }\end{array}$ & $6.2 \pm 1.5$ & $6.2 \pm 1.5$ & .98 \\
$\begin{array}{l}\text { MR imaging } \\
\quad \text { High SI in periventricular } \\
\quad \text { white matter }\end{array}$ & $0.65 \pm 1.3$ & $-0.12 \pm 1.4$ & $.045^{\mathrm{a}}$ \\
$\quad$ Gyral effacement & 3 & 0 & $<.001^{\mathrm{a}}$ \\
\hline
\end{tabular}

Note:-SI indicates signal intensity.

a Statically significant.

Pre stent CCT

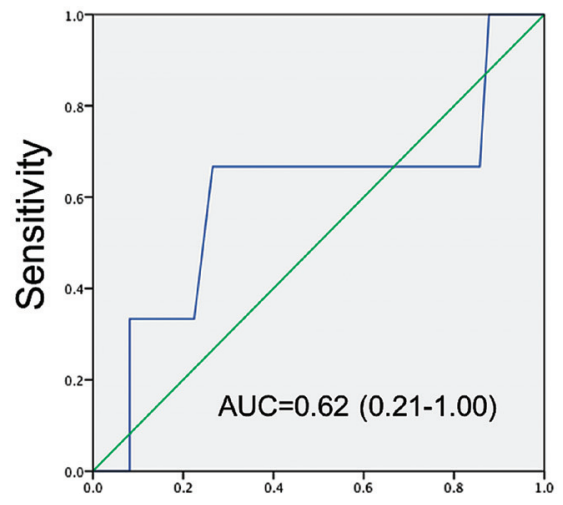

A

1-Specificity

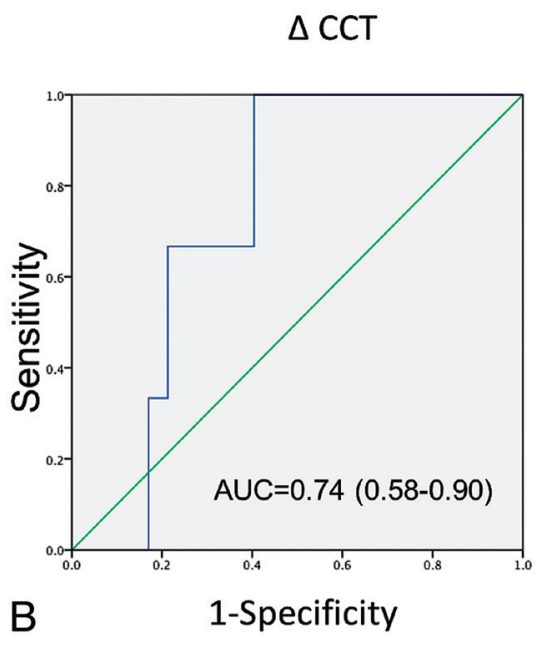

B

\section{RESULTS}

All procedures were successful. There were 15 patients in group A (hypoplastic or atretic TS) and 34 patients in group B (normal ipsilateral venous drainage). Patient characteristics are shown in Table 1. No significant differences in age, sex, degree of stenosis, incidences of prior stroke, or hypertension between the 2 groups were found. Peritherapeutic CCTs are listed in Table 2. The prestent CCTs of group A $(6.9 \pm 1.2)$ were longer than those of group B $(6.2 \pm 1.2)$, but they failed to achieve statistical significance. There were equivalent poststent CCTs between the 2 groups. However, the $\triangle \mathrm{CCT}$ was significantly shortened in group A $(0.65 \pm 1.3)$ compared with group B $(0.12 \pm 1.4)$. Figure 2 demonstrates the receiver operating characteristic analysis for both prestent CCT and $\triangle$ CCT versus HPS, respectively. The cutoff time of 7.1 seconds for prestent CCT predicted HPS in MR imaging with $67 \%$ sensitivity and $61 \%$ specificity. The cutoff time of 0.4 seconds for $\triangle$ CCT predicted HPS in MR imaging with $100 \%$ sensitivity and $40 \%$ specificity.

There were 3 patients in group A with increased signal intensities of white matter on MR images. One patient developed headache with nausea and vomiting 8 hours after the procedure (Table 3). A poststenting MR image demonstrated increased high signal intensity in the ipsilateral occipital subcortical white matter (Fig 3). Strict blood pressure control below $130 \mathrm{~mm} \mathrm{Hg}$ was implemented for 3 days, and the clinical follow-up was uneventful. None of the patients in group B showed abnormally high signal intensities of white matter or hyperperfusion syndrome clinically.

\section{DISCUSSION}

Regardless of the degree of stenosis of the ipsilateral TS, the CCTs of both groups returned to 6.2 seconds after treatment, consistent with the CCTs of normal populations in the literature. ${ }^{13}$ One previous study has also shown that the increased arterial flow after stent placement only shortened the circulation time in arterial ROIs, but not in venous ROIs. ${ }^{13}$ Therefore, it is likely that the prolonged CCT in group A before stent placement was caused by coexistent carotid stenosis and ipsilateral TS stenosis. The carotid stenosis caused impeded arterial flow superimposed on the stenotic venous outlet, and the overall venous outflow also decreased and led to prolonged CCT in group A. The differences in $\triangle \mathrm{CCT}$ between the 2 groups were likely caused in part by the normalization of prolonged CCT in the stenotic group.

In contrast to our results, Narita et $\mathrm{al}^{12}$ found shortening of the CCT after carotid stent placement. This is likely the result of the ROIs that were measured. Narita et al defined CCT by differences of time to maximal intensity between the cavernous portion of the internal carotid artery and the parietal vein. To assess the venous outflow, we used the internal carotid artery and in-

FIG 2. Receiver operating characteristic analysis of prestent CCT $(A)$ and $\Delta C C T(B)$. 
Table 3: Characteristics of 3 patients with high SI in the white matter in FLAIR sequences

\begin{tabular}{lclccccccc}
\hline Patient & Recent Infarct & HPS & Age $(\mathbf{y r})$ & Sex & Complications & Stenotic Degree & Pre-CCT (sec) & Post-CCT (sec) & $\Delta$ CCT (sec) \\
\hline 1 & No & Headache & 51 & F & No & $85 \%$ & 7.5 & 5.84 & 1.66 \\
2 & No & No & 79 & F & No & $77 \%$ & 5.5 & 5.03 & 0.47 \\
3 & No & No & 81 & M & No & $78 \%$ & 10.0 & 9.0 & 1.0 \\
\hline
\end{tabular}

Note:-SI indicates signal intensity.

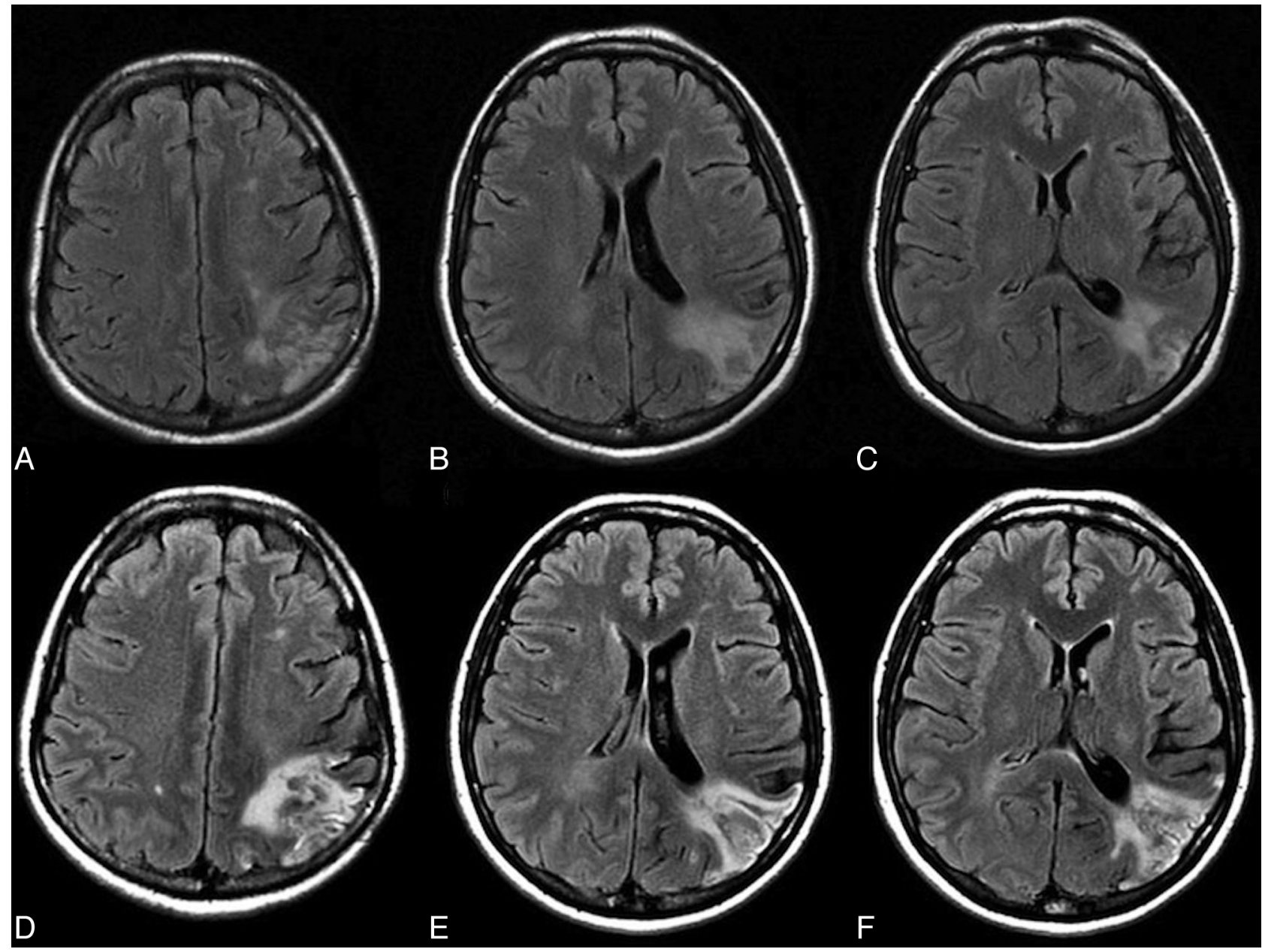

FIG 3. A 51 -year-old woman with $90 \%$ left internal carotid artery stenosis. Color-coded cerebral DSA demonstrated an ipsilateral atretic transverse sinus in Fig 1. A-C, Prestent MR imaging shows an old infarct in the occipital subcortical white matter. She had headache with nausea 8 hours after the procedure. $D-F$, MR imaging 12 hours after the procedure shows increased signal intensity over the left occipital subcortical white matter.

ternal jugular vein as reference points. A possible mechanism of unchanged CCT after stent placement is autoregulation of the brain occurring at the capillary level, which may have somehow neutralized the benefits of increased upstream arterial flow after stent placement, which could then not be detected at the downstream venous level.

The prolonged CCT before stent placement in the stenotic group indicated a greater degree of hemodynamic impairment. Given that the number of cases of poor collateral circulation did not differ significantly between the 2 groups (13\% versus $18 \%$ ), we speculated that inadequate venous drainage in the stenotic group might have been associated with a dysfunction of autoregulation. Previous literature has shown good correlations between angiography CCT and MTT from perfusion studies. ${ }^{16,17}$ Longer MTT indicated pre-existing hemodynamic impairment and predisposed the patient to HPS. ${ }^{18,19}$
The high signal intensity of subcortical white matter on FLAIR imaging represented edematous change and implied impaired dysautoregulation, likely potentiated by the venous stasis. If one reviewed the 3 patients with abnormal MR imaging findings, no incidences of contralateral stenosis or poor collaterals were seen, their mean age was 70 years, and strict postoperation blood pressure control was also performed. ${ }^{7,17}$ Thus, shortened $\Delta$ CCT combined with venous stenosis seems to cause an increase in cerebral blood flow. Note, however, that blood-brain barrier breakdown is another possible cause, which was not explored in our study. ${ }^{11}$

Asymmetry of the dural sinus is common and does not generally lead to clinical symptoms. Once increased cerebral blood flow has developed following successful recanalization, however, better venous drainage may be required to relieve the sudden increase in cerebral blood flow. All our patients who experienced HPS had severe venous outlet anomalies. Perhaps hyperperfusion

AJNR Am J Neuroradiol 35:1132-36 Jun 2014 www.ajnr.org 1135 
following carotid stent placement in patients with ipsilateral poor venous drainage may follow a similar mechanism as that found in sinus thrombosis with hemorrhage complications: increased venous pressure from acute thrombosis or outlet obstruction precipitating venous hypertension and congestion. ${ }^{17,20,21}$

There were several limitations in our study. First, our study population was fairly small due to the low incidence of hyperperfusion, and larger scale studies are needed to confirm our hypothesis. Second, the determination of time to maximal intensity in venous ROIs is vulnerable to waveform changes. The difference between the arrival time of arterial and venous ROIs might be a better surrogate to designate CCT. Unfortunately, this parameter was not available with the current version of the software. Although the mean $\Delta$ CCTs were significantly different in the 2 groups, the wide overlapping ranges of prestenting CCT and $\Delta$ CCT in both groups handicapped these parameters as predictors for HPS in clinical practice. The definition of white matter change was subjective and thus was challenging for cases with mild venous congestion. Future volumetric MR images can potentially quantify the measurement. ${ }^{22}$ Finally, the autoregulation of cerebral perfusion after stent placement was dynamic; the most appropriate timing of examinations to predict hyperperfusion deserves more detailed investigation.

\section{CONCLUSIONS}

Ipsilateral hypoplastic TSs were associated with prolonged CCT before stent placement and greatly shortened CCT after stent placement. Inadequate venous drainage might play a role in the development of poststenting hyperperfusion syndrome.

Disclosures: Wan-Yuo Guo-RELATED: research agreement between Taipei Veterans General Hospital and Siemens Healthcare*; UNRELATED: Payment for Manuscript Preparation: based on the contract between the hospital and sponsors; certain compensation is given after a paper has been accepted for publication. All processes are under the supervision of the Hospital administration following governmental regulation. * Money paid to the institution.

\section{REFERENCES}

1. Chen YH, Lin MS, Lee JK, et al. Carotid stenting improves cognitive function in asymptomatic cerebral ischemia. Int $J$ Cardiol 2012;157:104-07

2. Lal BK. Cognitive function after carotid artery revascularization. Vasc Endovascular Surg 2007;41:5-13

3. Mas JL, Chatellier G, Beyssen B, et al. Endarterectomy versus stenting in patients with symptomatic severe carotid stenosis. $N$ Engl J Med 2006;355:1660-71

4. Sixt S, Rastan A, Schwarzwalder U, et al. Long term outcome after balloon angioplasty and stenting of subclavian artery obstruction: a single centre experience. Vasa 2008;37:174-82

5. Meyers PM, Higashida RT, Phatouros CC, et al. Cerebral hyperperfusion syndrome after percutaneous transluminal stenting of the craniocervical arteries. Neurosurgery 2000;47:335-43, discussion 43-45

6. Rezende MT, Spelle L, Mounayer C, et al. Hyperperfusion syndrome after stenting for intracranial vertebral stenosis. Stroke 2006;37:e12-14

7. Ogasawara K, Sakai N, Kuroiwa T, et al. Intracranial hemorrhage associated with cerebral hyperperfusion syndrome following carotid endarterectomy and carotid artery stenting: retrospective review of 4494 patients. J Neurosurg 2007;107:1130-36

8. Ascher E, Markevich N, Schutzer RW, et al. Cerebral hyperperfusion syndrome after carotid endarterectomy: predictive factors and hemodynamic changes. J Vasc Surg 2003;37:769-77

9. Abou-Chebl A, Yadav JS, Reginelli JP, et al. Intracranial hemorrhage and hyperperfusion syndrome following carotid artery stenting: risk factors, prevention, and treatment. J Am Coll Cardiol 2004;43:1596-601

10. Kawamata T, Okada Y, Kawashima A, et al. Postcarotid endarterectomy cerebral hyperperfusion can be prevented by minimizing intraoperative cerebral ischemia and strict postoperative blood pressure control under continuous sedation. Neurosurgery 2009;64:447-53, discussion 53-54

11. Ivens S, Gabriel S, Greenberg G, et al. Blood-brain barrier breakdown as a novel mechanism underlying cerebral hyperperfusion syndrome. J Neurol 2010;257:615-20

12. Narita $S$, Aikawa $H$, Nagata $S$, et al. Intraprocedural prediction of hemorrhagic cerebral hyperperfusion syndrome after carotid artery stenting. J Stroke Cerebrovasc Dis 2013;22:615-19

13. Lin CJ, Hung SC, Guo WY, et al. Monitoring peri-therapeutic cerebral circulation time: a feasibility study using color-coded quantitative DSA in patients with steno-occlusive arterial disease. AJNR Am J Neuroradiol 2012;33:1685-90

14. Strother CM, Bender F, Deuerling-Zheng Y, et al. Parametric color coding of digital subtraction angiography. AJNR Am J Neuroradiol 2010;31:919-24

15. Greitz T. A radiologic study of the brain circulation by rapid serial angiography of the carotid artery. Acta Radiol Suppl 1956:1-123

16. Aikawa H, Kazekawa $\mathrm{K}$, Tsutsumi $\mathrm{M}$, et al. Intraprocedural changes in angiographic cerebral circulation time predict cerebral blood flow after carotid artery stenting. Neurol Med Chir (Tokyo) 2010;50:269-74

17. Lee KW, Tsai FY, Cheng CY. Venous hypertension may be a factor in aneurysmal rupture. Neuroradiol J 2013;26:311-14

18. Rim NJ, Kim HS, Shin YS, et al. Which CT perfusion parameter best reflects cerebrovascular reserve?: correlation of acetazolamidechallenged CT perfusion with single-photon emission CT in Moyamoya patients. AJNR Am J Neuroradiol 2008;29:1658-63

19. Hosoda K, Kawaguchi T, Shibata Y, et al. Cerebral vasoreactivity and internal carotid artery flow help to identify patients at risk for hyperperfusion after carotid endarterectomy. Stroke 2001;32:1567-73

20. Tsai FY, Nguyen B, Lin WC. Endovascular procedures for cerebrovenous disorders. Acta Neurochir Suppl 2008;101:83-86

21. Tsai FY, Wang AM, Matovich VB, et al. MR staging of acute dural sinus thrombosis: correlation with venous pressure measurements and implications for treatment and prognosis. AJNR Am J Neuroradiol 1995; 16:1021-29

22. Artzi M, Aizenstein O, Jonas-Kimchi T, et al. FLAIR lesion segmentation: application in patients with brain tumors and acute ischemic stroke. Eur J Radiol 2013;82:1512-18 